\title{
Investigation of paper aging assessment by furan concentration monitoring
}

\begin{abstract}
Transformers are the vital section of transmission and distribution electrical network. In addition, they are the most expensive investment in the network. Therefore, they should be evaluated for proper working and monitor regularly to assess normal condition and life expectancy. Obviously, the health of transformer is strictly depending on transformer's insulation conditions, oil and paper, that both of them degrade along the time and are temperature dependent. There are some diagnostic methods to evaluate oil and paper conditions by considering of degradation byproducts. But after all, only furanic compounds are unique indicators of paper aging assessment of transformers. Hence, this article attempts to illustrate the paper thermal degradation mechanism and temperature depending of furans concentration in oil and specially relationship between furan concentration (in our purpose 2furaldehyde or 2 FAL caused by overheating) with degree of polymerization, paper strength and life expectancy of transformers.
\end{abstract}

Keyword: Transformer; Insulation; Degradation; Furans 